\title{
Comprehensive Diagnostic Criteria for MELAS Syndrome; a Case Study Involving an Elderly Patient With MT-TWm.5541C>T Mutation
}

\section{Gang Wu}

Taizhou Hospital of Zhejiang Province

\section{Yuguang Shen}

Taizhou Hospital of Zhejiang Province

Feng Zhu

Taizhou Hospital of Zhejiang Province

Weiwei Tao

Taizhou Hospital of Zhejiang Province

\section{Yuanlin Zhou}

Taizhou Hospital of Zhejiang Province

Shaofa Ke

Taizhou Hospital of Zhejiang Province

Huihua Jiang ( $\nabla$ jianghh@enzemed.com )

Taizhou Hospital of Zhejiang Province affiliated to Wenzhou Medical University

\section{Case Report}

Keywords: MELAS, recurrent stroke, MRI, lactate, MT-TW mutation, case report

Posted Date: October 29th, 2021

DOl: https://doi.org/10.21203/rs.3.rs-961557/v1

License: (c) (1) This work is licensed under a Creative Commons Attribution 4.0 International License. Read Full License 


\section{Abstract}

Background: Mitochondrial encephalopathy, lactic acidosis, and stroke-like episodes (MELAS) syndrome is a matrilineal hereditary multi-system disease due to mutations of mitochondrial DNA. Though the initial diagnostic criteria prevail till nowadays, which take a range of clinical phenotypes into consideration including clinical onset after the age of 40 , a confirmative diagnostic standard for MELAS is still needed.

Case presentation: A 71-year-old female patient presented with recurrent stroke to our hospital. Magnetic resonance imaging showed a cerebral gyrus-like area with hyperintensity in the parietal-occipital lobe on DWI and this area expanded with disease progression. The Lac/Lip peaks were significantly inverted in the MRS imaging. Nucleic acid sequencing showed a MT-TWm.5541C>T mutation, and the mutation rate of the blood sample was $12.86 \%$. The patient had a 6-year history of type 2 diabetes. The patient experienced recurrent stroke and was given coenzyme Q10 capsules and idebenone tablets to improve metabolism, vitamin B to nourish nerves, edaravone to scavenge oxygen free radicals, and oxcarbazepine to manage seizures. At 1-month follow-up, her condition was stable.

Conclusion: Patients with MELAS syndrome have complex clinical manifestations. Our data demonstrated that, for patients with atypical cerebral infarction and suspected MELAS syndrome, gene sequencing and muscle biopsy should be completed in time, which may serve as a reference for the diagnostic criteria of MELAS syndrome.

\section{Background}

MELAS syndrome is a matrilineal hereditary disease involving multiple systems due to mutations in the mitochondrial DNA. It is clinically characterized by stroke-like episodes, focal or generalized seizures, recurrent migraine-like headache, vomiting, short stature, hearing loss, and muscle weakness ${ }^{1,2}$. Although studies have shown that MELAS syndrome occurs in patients over 40 years old and clinical symptoms vary between patients, which are the key elements for clinical diagnosis. However, a definite objective diagnostic criterion is still in demand. In this case report, we demonstrated clinical, laboratory, and imaging results of a 71-year-old female patient who developed MELAS syndrome. These findings might facilitate the compilation of a more comprehensive diagnostic standard for MELAS syndrome.

\section{Case Presentation}

A 71-year old, 1.47-meter tall woman was admitted to Taizhou Hospital of Zhejiang province 28 hours after the onset of sudden weakness in her right upper limb. The patient had a history of type 2 diabetes, spasmodic torticollis, cerebral infarction, and left clavicle fracture. The patient had no history of mental retardation or cognitive impairment. In her childhood, youth, and middle age, she appeared to be healthy except for the short stature. Spasmodic torticollis was observed at the age of 62 and type 2 diabetes at the age of 65 . Glycemic control measures were taken but the effect was unknown. At the age of 68 , she 
was diagnosed with cerebral infarction and discharged with left-limb weakness remained and ambiguity after symptomatic treatment. The patient had no history of frequent headaches (Supplementary material 1). The drugs she took included clopidogrel, atorvastatin, tiapride hydrochloride, and metformin tablets. In addition, her mother died from an unknown cause in her youth.

At admission, physical examination showed that blurred speech, while her muscle strength of both upper

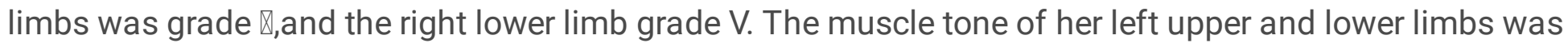
elevated, the tendon reflex of extremities was active, and the Babinski sign was negative on both sides. The NIHSS score was 5. Cranial magnetic resonance imaging (MRI) showed abnormal signals in the left postcentral gyrus and acute cerebral infarction or encephalitis was suspected (Figure 1. A1 to A4). Biochemical tests showed hyperglycemia $(11.7 \mathrm{mmol} / \mathrm{L})$, a high level of glycosylated hemoglobin $(6.5 \%)$ and serum lactic acid $(5.1 \mathrm{mmol} / \mathrm{L}$, normal value $0.5-1.6 \mathrm{mmol} / \mathrm{L})$. In addition, CT angiography showed no significant stenosis or plaques in intracranial vessels. Transthoracic echocardiography excluded valvular lesions, thrombi, or excrescence signals in cardiac cavities. 24-hour dynamic electrocardiogram examination (Holter monitor) excluded atrial fibrillation or arrhythmia due to other causes. Consequently, the patient was diagnosed with ischemic stroke on admission and managed with clopidogrel tablets to prevent platelet aggregation.

Despite proactive treatment, the patient showed deterioration in her condition. She could not lift her right upper or lower limb, and had paroxysmal convulsions and increased muscle tone in the right upper limb. The patient gradually manifested with aphasia, blurred vision, disorientation, and even perturbed consciousness. Neurological assessment showed a NIHSS score of 15. Emergent CT scan showed subacute cerebral infarction in the left parietal lobe, with no lesion enlargement or hemorrhage. To further clarify the character of the lesion, MR scan with dynamic enhancement (3.0T) and spectroscopy MRS (3.0T) were completed. A larger area of gyrus-like hyperintensity in the left parietal-occipital lobe was observed on DWI. Linear enhancement of the gyrus-like lesion was also detected in contrast-enhanced scan with no thickening of adjacent meninges (Figure1.B1-B4). MRS analysis showed a slightly decreased N-acetylaspartic acid (NAA) peak and an inverted Lac/Lip peak in the focal area (Figure 2). The concentration of blood glucose fluctuated between 4.4 and $10.4 \mathrm{mmol} / \mathrm{L}$ before the deterioration of her condition. Given the history of cerebral infarction, abnormal gyrus-like signals in the cerebral lobe, inverted Lac/Lip peak on MRS, and increased lactic acid in the blood, MELAS syndrome were suspected. The patient was then given coenzyme Q10 capsules and idebenone tablets to improve metabolism, vitamin $B$ to nourish nerves, edaravone to scavenge oxygen free radicals, and oxcarbazepine to manage seizures.

To test our suspicion of MELAS syndrome, DNA was extracted from white blood cells of the patient and her two daughters after obtaining their written informed consent. Sequencing of mitochondrial genes showed that this patient had $12.86 \%$ MT-TW m.5541C $>$ T mutation. Her elder daughter had a suspected heterogeneous mutation, while the younger daughter did not show any mutation (Figure 3). 
To rule out mutations of nuclear genes that might impact the respiratory chain or mitochondrial functions, nuclear genes were sequenced. It was found that there were no mutations in nuclear genes, such as the cytochrome $\mathrm{C}$ oxidase (COX) and succinate dehydrogenase (SDH), which impact mitochondrial functions. Unlike the younger daughter, the patient and her elder daughter had a heterozygous missense mutation of SLC30A8_ex8c.973C > T, which is reported to be associated with noninsulin dependent diabetes mellitus (NIDDM) ${ }^{3}$ (Supplementary material 2).

During the follow-up after one month, the condition of the patient was relatively stable with paroxysmal convulsions in the right upper limb being relieved and responsive to oral stimuli within the family, but weakness of the limbs was still present. The NIHSS score was 8 . After consulting with our specialist, the family decided to continue rehabilitation therapy in the local hospital.

\section{Discussion}

MELAS syndrome is a rare mitochondrial disease caused by mutations in the mitochondrial DNA. It is characterized by stroke-like episodes leading to hemiplegia, hemianopsia, or cortical blindness. Other common features include focal or generalized seizures, recurrent migraine-like headaches, vomiting, short stature, hearing loss, and muscle weakness ${ }^{1,2}$. Stroke-like episodes typically present with acute onset of neurological symptoms in patients with MELAS syndrome with hyperintensities on DWI. Upon brain imaging, lesions are generally focal complying with the distribution of blood vessels. Values of apparent diffusion coefficient (ADC) do not necessarily decrease. At the acute stage, MRI signals may migrate, fluctuate, or even disappear. These changes are faster and more common than in typical ischemic stroke patients ${ }^{4}$. The patient we reported here had recurrent stroke, changeable foci, and seizures in the course of the disease. Our imaging results revealed lobar lesions that did not conform to the distribution of blood vessels. Other complementary tests did not show cerebrovascular lesions or possible cardiothrombotic diseases. Therefore, this patient is very likely to develop MELAS syndrome.

Due to the complex nature of pathogenesis and clinical manifestations, unified comprehensive diagnostic criteria have not been reached yet, rendering a likelihood of being missed or misdiagnosed for certain patients with MELAS syndrome. The initial diagnostic criteria for MELAS syndrome include: stroke-like episodes before the age of 40 , encephalopathy characterized by seizures or dementia, and signs of mitochondrial myopathy evidenced by blood lactic acidosis or broken red fibers in skeletal muscle biopsy. Another two tests can be tentatively used to confirm the diagnosis, including normal early psychomotor development, recurrent headache or repeated vomiting ${ }^{5}$. Subsequent studies encompassed a wider range of phenotypes for MELAS syndrome diagnosis, a good example is clinical onset after the age of 40 years ${ }^{6-8}$. In 2012, the Japanese MELAS Research Group Committee adopted the following diagnostic criteria requiring at least two signs of clinical criteria A - consisting of headache with vomiting, seizures, hemiplegia, cortical blindness and acute focal lesions revealed by neuroimaging, in combination with at least two positive tests in laboratory criteria B - consisting of high levels of plasma 
or cerebrospinal fluid (CSF) lactate, mitochondrial abnormalities in muscle biopsy, and MELAS-related gene mutations ${ }^{9}$.

The 71-year-old patient presented with recurrent cerebral infarction. However, no cardiothrombotic causes, such as atrial fibrillation or vulnerable plaques of the carotid artery, were found. The condition of this patient deteriorated though conventional treatment has been administered. Surprisingly, it was stabilized after taking coenzyme Q10 and vitamin B, both of which are used to improve metabolism. This patient presented with stroke-like episodes, aphasia, seizure, acute gyrus-like lesions in the parietal-occipital lobe on MRI, increased blood lactic acid, and a history of relatively short stature and spasmodic torticollis, which led to the suspicion of MELAS syndrome. Gene sequencing showed a 12.86\% MT-TWm.5541C>T mutation in mitochondrial genes of leukocytes. Nuclear gene sequencing showed no mutations associated with mitochondrial dysfunction. A heterozygous mutation in the SLC30A8 gene was found, but it is associated with NIDDM instead of mitochondrial functions.

A number of mitochondrial tRNA mutations can cause MELAS syndrome. $80 \%$ of MELAS patients are associated with the $\mathrm{m} .3243 \mathrm{~A}>\mathrm{G}$ mutation, whereas $10 \%$ associated with the $\mathrm{m} .3271 \mathrm{~T}>\mathrm{C}$ mutation ${ }^{10}$. Interestingly, the patient reported in the present study showed MT-TWm.5541C>T mutation in mitochondrial gene sequencing. Yarham et al constructed a scoring system for mutation pathogenicity assessment which uses multiple weighted criteria including a series of molecular, functional, and genetic data ${ }^{11}$. Using an updated version of the scoring system, Blakely et al confirmed that the MT-TW m.5541C>T mutation was a "confirmed pathogenic mutation"12. Though this mutation can lead to the occurrence of clinical MELAS syndrome, there are still few reports on this mutation.

In theory, there is a threshold, also known as the threshold effect, of mtDNA mutations beyond which certain tissues or organs begin to dysfunction. However, different tissues have discrete thresholds based on their degree of energy dependence. The brain is highly dependent on energy and thus has a relatively low threshold. A male patient with a mtDNA mutation m.5541C>T showed MELAS symptoms with a mutation rate of $84 \%$ in skeletal muscles, but his mother who had a mutation rate of $51 \%$ in skeletal muscles did not show MELAS symptoms ${ }^{12}$. In the patient reported in the present study, gene sequencing showed a mutation rate of $12.86 \%$ in leucocytes, which was relatively lower than the abovementioned rates, but she had MELAS symptoms. On one hand, gene sequencing was performed on white blood cells, not muscles in the present study. On the other hand, "heterogenization" of the mtDNA disorder signifies different distribution of the mutated mtDNA in diverse tissues. For example, the mutation rate of mitochondrial gene detected in muscles is often higher than that in the blood ${ }^{12,13}$. Furthermore, individual brain tissues have different tolerance to metabolic needs, especially if there are underlying metabolic diseases that influence brain metabolism ${ }^{14}$. The patient in this study carried a SLC30A8_ex8c.973C>T missense mutation associated with NIDDM and was diagnosed with type 2 diabetes. Other mutations may result in changes in the activity or structure of other enzymes or proteins that impact brain metabolism. To date, however, specific pathophysiological mechanisms still need to be further investigated. 
Due to the lack of cooperation from family members of the patient and the cost of gene sequencing, the patient did not allow to perform mitochondrial gene sequencing and biopsy on skeletal muscles. However, recurrent stroke-like episodes, MRI findings, elevated lactate in the blood, and inverted Lac/Lip peak on MRS render us to diagnose the patient with MELAS syndrome.

\section{Conclusion}

In conclusion, clinical manifestations of MELAS syndrome are complex and imaging findings are dynamic. For patients with atypical cerebral infarction and suspected MELAS syndrome, gene sequencing and muscle biopsy should be completed in time. The patient in our study showed symptoms consistent with MELAS syndrome, but her diagnosis was not confirmed based on the strict diagnostic criteria. Currently, the patient can only be diagnosed with a mimic-MELAS syndrome. With the in-depth understanding of MELAS syndrome and the development of new detection technologies, more patients of mimic-MELAS syndrome might be reported. Our findings might inform the development of more reasonable diagnostic criteria for MELAS syndrome.

\section{Abbreviations}

MELAS: Mitochondrial encephalopathy, lactic acidosis, and stroke-like episodes syndrome; MRI: Magnetic resonance imaging; DWI: Diffusion weighted imaging; ADC: Apparent diffusion coefficients; NIHSS: National institutes of health stroke scale; NAA: N-acetylaspartic acid; COX: Cytochrome C oxidase; SDH: Succinate dehydrogenase; NIDDM: Non-insulin dependent diabetes mellitus

\section{Declarations}

\section{Ethical approval and consent to participate}

This report has been approved by the Ethical Review Board of Taizhou Hospital. The patient and her family agreed to participate in this study.

\section{Consent for publication}

Written informed consent was obtained from the patient and her family to publish this manuscript.

\section{Availability of data and materials}

All data are available from the corresponding author upon reasonable request.

\section{Competing interests}

The authors declare that they have no competing interests.

\section{Funding}


Not applicable.

\section{Authors' contributions}

Conceptualization: Huihua Jiang; Visualization: Gang Wu, Yuguang Shen, Feng Zhu, Weiwei Tao; Writing - original draft: Gang Wu, Yuguang Shen, Feng Zhu; revision and editing: Huihua Jiang, Yuanlin Zhou, Shaofa Ke. Approval of final manuscript: all authors.

\section{Acknowledgements}

Not applicable.

\section{References}

1. Koenig MK, Emrick L, Karaa A, Korson M, Scaglia F, Parikh S, Goldstein A. Recommendations for the Management of Strokelike Episodes in Patients With Mitochondrial Encephalomyopathy, Lactic Acidosis, and Strokelike Episodes. JAMA Neurol 2016,73(5):591-4. https://doi.org/10.1001/jamaneurol.2015. 5072.

2. El-Hattab AW, Adesina AM, Jones J, Scaglia F. MELAS syndrome: Clinical manifestations, pathogenesis, and treatment options. Mol Genet Metab 2015,116(1-2):4-12. https://doi.org/10.1016/j.ymgme.2015.06.004

3. Sladek R, Rocheleau G, Rung J, et al. A genome-wide association study identifies novel risk loci for type 2 diabetes. Nature 2007, 445(7130):881-885. https://doi.org/10.1038/nature05616

4. Malhotra K, Liebeskind DS. Imaging of MELAS. Curr Pain Headache Rep 2016, 20(9):54. https://doi.org/10.1007/s11916-016-0583-7

5. Hirano M, Ricci E, Koenigsberger MR, Defendini R, Pavlakis SG, DeVivo DC, DiMauro S, Rowland LP. Melas: an original case and clinical criteria for diagnosis. Neuromuscul Disord 1992, 2(2):125-135. https://doi.org/10.1016/ 0960-8966(92)90045-8

6. Dimauro S, Tay S, Mancuso M. Mitochondrial encephalomyopathies: diagnostic approach. Ann N Y Acad Sci 2004,1011:217-231. https://doi.org/10.1196/annals.1293. 022

7. Dickerson BC, Holtzman D, Grant PE, Tian D. Case records of the Massachusetts General Hospital. Case 36-2005. A 61-year-old woman with seizure, disturbed gait, and altered mental status. N Engl J Med 2005, 353(21):2271-2280. https://doi.org/ 10.1056/NEJMcpc059032

8. Fang GL, Zheng Y, Zhang YX. Mitochondrial encephalomyopathy with lactic acidosis and stroke-like episodes in an older adult mimicking cerebral infarction: a Chinese case report. Clin Interv Aging 2018,13:2421-2424. https://doi.org/10.2147/CIA. S186636

9. Yatsuga S, Povalko N, Nishioka J, Katayama K, Kakimoto N, Matsuishi T, Kakuma T, Koga Y. MELAS: a nationwide prospective cohort study of 96 patients in Japan. Biochim Biophys Acta 2012, 1820(5):619-624. https://doi. org/10.1016/j.bbagen.2011.03.015 
10. Ikeda T, Osaka H, Shimbo H, Tajika M, Yamazaki M, Ueda A, Murayama K, Yamagata T. Mitochondrial DNA 3243A>T mutation in a patient with MELAS syndrome. Hum Genome Var 2018, 5:25. https://doi.org/10.1038/s41439-018-0026-6

11. Yarham JW, Al-Dosary M, Blakely EL, Alston CL, Taylor RW, Elson JL, McFarland R. A comparative analysis approach to determining the pathogenicity of mitochondrial tRNA mutations. Hum Mutat 2011, 32(11):1319-1325. https://doi.org/ 10.1002/humu.21575

12. Blakely EL, Yarham JW, Alston CL, Craig K, Poulton J, Brierley C, Park SM, Dean A, Xuereb JH, Anderson $\mathrm{KN}$ et al. Pathogenic mitochondrial tRNA point mutations: nine novel mutations affirm their importance as a cause of mitochondrial disease. Hum Mutat 2013, 34(9):1260-1268. https://doi.org/ 10.1002/humu.22358

13. El-Hattab AW, Almannai M, Scaglia F. MELAS. In: Adam MP, Ardinger HH, Pagon RA, et al., eds. GeneReviews ${ }^{\circledR}$. Seattle (WA): University of Washington, Seattle; February 27, 2001.

14. Li W, Zhang W, Li F, Wang C. Mitochondrial genetic analysis in a Chinese family suffering from both mitochondrial encephalomyopathy with lactic acidosis and stroke-like episodes and diabetes. Int J Clin Exp Pathol 2015, 8(6):7022-7027.

\section{Figures}

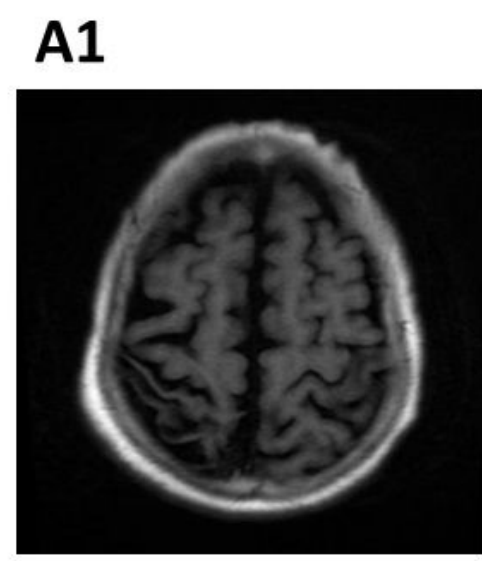

B1

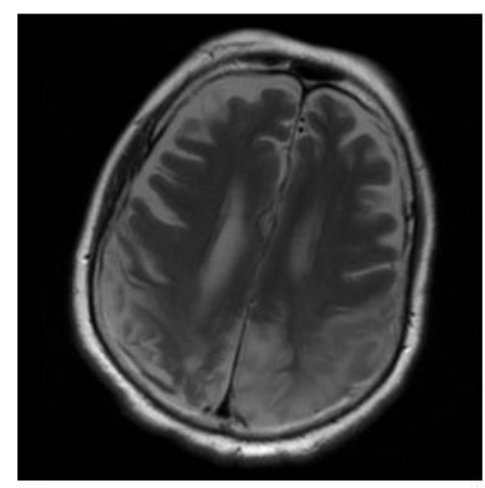

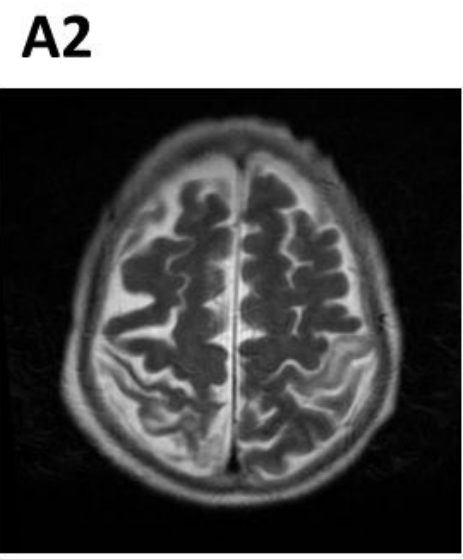

B2

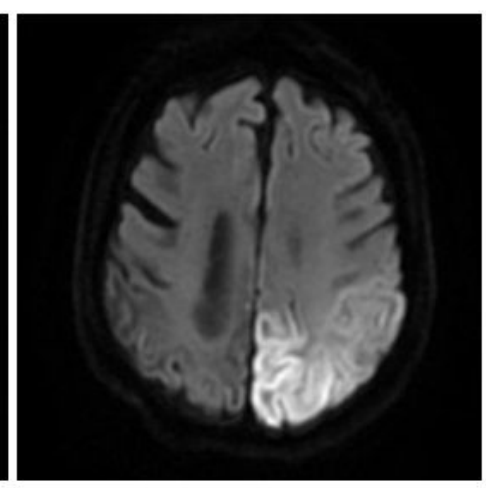

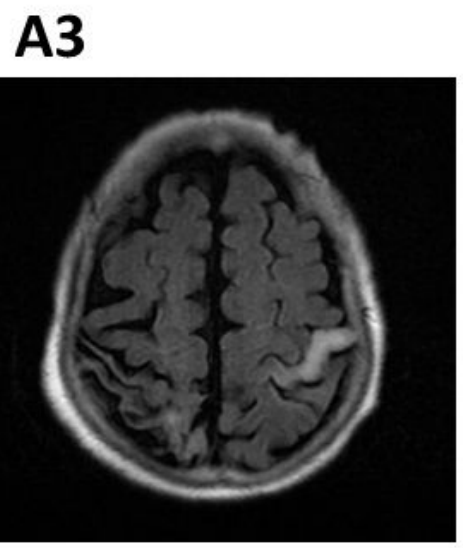

B3

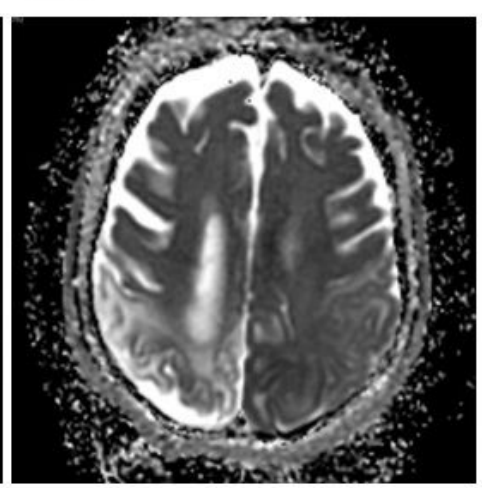

A4

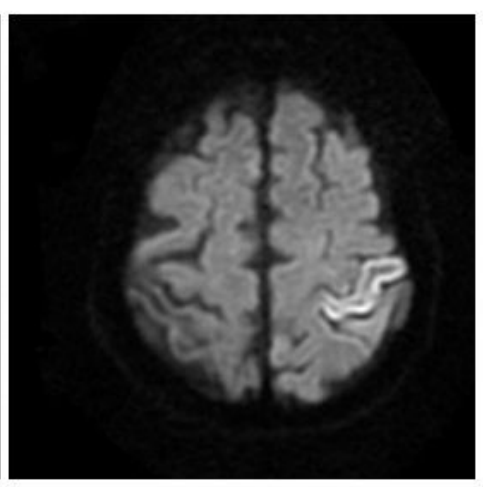

B4

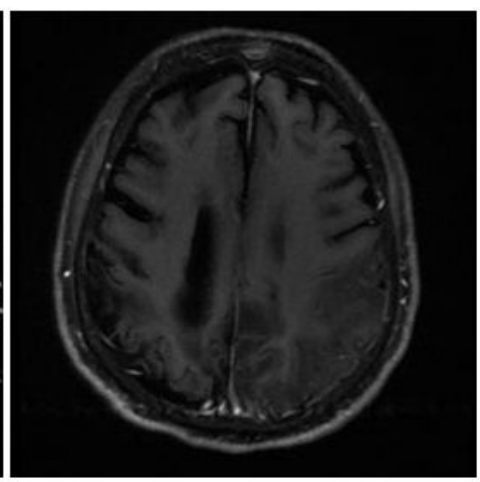

Figure 1 
MRI changes during hospital stay. A1-A4 showed an abnormal signal in the left postcentral gyrus on T1, T2, FLAIR, and DWI, respectively, at admission. B1-B4 demonstrated a larger lesion on T2, DWI, ADC, and T1 with dynamic enhancement, respectively, when the condition of the patient deteriorated. An expanded lesion in the left parietal-occipital lobe was found after conservative treatment of ischemic stroke. ADC and DWI showed limited diffusion in the left parietal-occipital lobe. T1 enhancement showed linear enhancement of the local gyri with no meningeal thickening in adjacent meninges. ADC; apparent diffusion coefficients.

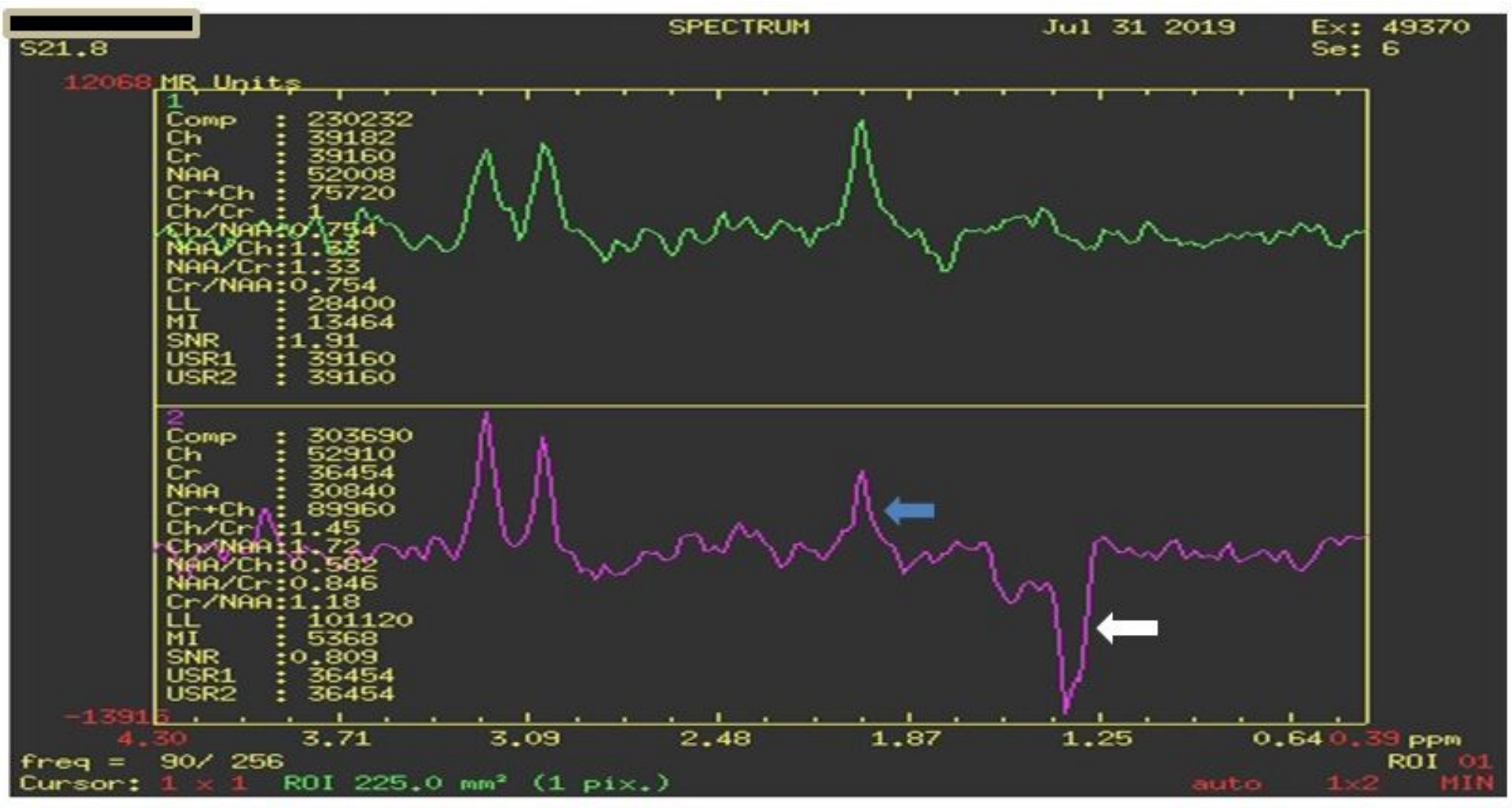

\section{Figure 2}

MR spectroscopy demonstrated an increase in lactate, presented as an inversed Lac/Lip peak at 1.3 (white arrow), a slight decrease in N-acetylaspartic acid (NAA), presented as a peak at 2.05 (blue arrow). 


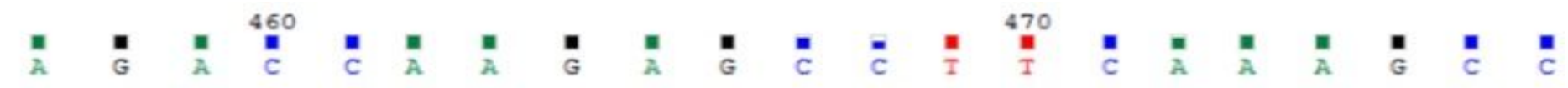
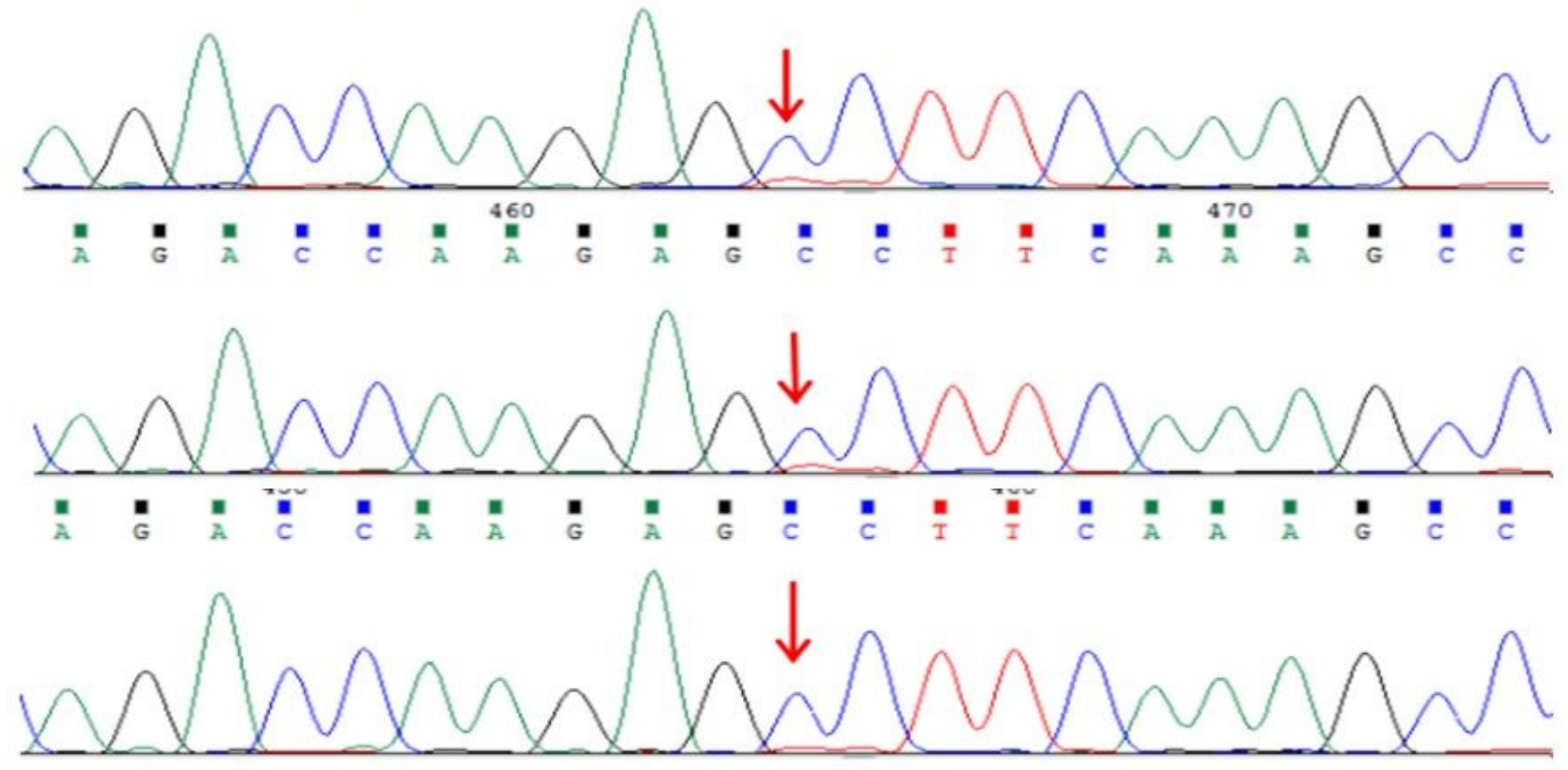

Figure 3

Gene sequencing of the MTTW revealed the m.5541C>T mutation. Sequencing chromatogram of the MTTW gene showed heteroplasmic m.5541C>T mutation in the patient (upper), suspected heterogeneous mutation in her elder daughter (middle) and no such mutation in her younger daughter (lower).

\section{Supplementary Files}

This is a list of supplementary files associated with this preprint. Click to download.

- Supplementarymaterial1and2.docx

- CAREchecklistEnglish2013.pdf 